\title{
Apolipoprotein E genotype related differences in brain lesions of multiple sclerosis
}

Franz Fazekas, Siegrid Strasser-Fuchs, Helena Schmidt, Christian Enzinger, Stefan Ropele, Anita Lechner, Erich Flooh, Reinhold Schmidt, Hans-Peter Hartung

\begin{abstract}
Objectives-Clinical reports have speculated on a more severe course of multiple sclerosis in patients with the apolipoprotein $\mathrm{E}$ (apoE) 84 allele. As this could be reflected by differences in the severity of tissue damage MRI was used to obtain further support for a disease modifying effect of the apoE genotype.

Methods-Brain MR scans of 83 patients (mean age 35.5 (SD 9.5 years) who participated in a cross sectional study on the distribution of genotype patterns in multiple sclerosis. The total lesion load on proton density weighted (T2-LL) and T1 weighted scans (T1-LL) obtained with conventional spin echo sequences at $1.5 \mathrm{~T}$ was measured. A "black hole" ratio ((T1LL/T2-LL) $\times 100$ ) was also calculated. This indicates the proportion of multiple sclerosis lesions with more severe tissue damage and may reflect disease aggressiveness or quality of repair.

Results-Patients with the apoE- $83 / \varepsilon 4$ genotype $(n=19)$ showed a nonsignificantly greater T2-LL (16.0 (SD 14.0) $\left.\mathrm{cm}^{3}\right)$ than patients with the $\varepsilon 2 / \varepsilon 3(\mathrm{n}=11$; $\left.13.3(9.5) \mathrm{cm}^{3}\right)$ or the $\varepsilon 3 / \varepsilon 3$ genotype $(\mathrm{n}=49$; $\left.9.4(\mathrm{SD} 9.2) \mathrm{cm}^{3}\right)$. Both the T1-LL (2.6 (SD 3.3) $v 1.6\left(\mathrm{SD} \mathrm{2.4)}\right.$ and $1.2(\mathrm{SD} 3.0) \mathrm{cm}^{3}$; $\mathrm{p}=0.04)$ and the black hole ratio (14.3 SD 11.9) $v 7.4$ (SD 9.3) and 8.4 (SD 13.3)\%; p=0.02), however, were significantly higher in $\varepsilon 3 / \varepsilon 4$ patients. Similar differences were seen when comparing patients with at least one $\varepsilon 4$ allele with the remainder of the group.

Conclusions-These data support speculations on a modulation of multiple sclerosis severity by the apoE genotype which can be attributed to more extensive tissue destruction or less efficient repair in carriers of the $\varepsilon 4$ allele.

(F Neurol Neurosurg Psychiatry 2000;69:25-28)
\end{abstract}

Keywords: multiple sclerosis; apolipoprotein E genotype; magnetic resonance imaging

Multiple sclerosis is the most frequent neurological autoimmune disorder and the leading cause of serious neurological disability in early adulthood. Prevalence rates of multiple sclerosis vary substantially throughout the world and this has been attributed to environmental and genetic factors. ${ }^{1-3}$ However, genetic factors may not only influence a person's susceptibility for multiple sclerosis but can also be expected to modulate the course of the disease. ${ }^{45}$ In this context the apolipoprotein E genotype could play some part.

Apolipoprotein E (apoE) is a polymorphic plasma glycoprotein that transports cholesterol and other lipids. ${ }^{6}$ The gene for apoE is located on chromosome 19 and is highly polymorphic. The three most common alleles are $\varepsilon 2, \varepsilon 3$, and $\varepsilon 4$, which encode the three main isoforms of apoE: E2, E3, and E4. In various cell lines apoE3 has been shown to increase the growth and branching of neurites, whereas apoE4 was found to have the opposite effect. ${ }^{7}$ At least equally important in the context of multiple sclerosis, there have been several reports indicating that apoE is involved in lipid redistribution after demyelination. ${ }^{8}$ Therefore, an isoform specific differential effect of apoE may not only affect the preservation of axons but also the process of remyelination. Accordingly, first reports based on clinical assessment of severity of disease showed some trend towards a more aggressive course of multiple sclerosis in apoE $\varepsilon 4$ carriers. ${ }^{9}{ }^{10}$ This prompted us to use brain MRI for further study of this hypothesis.

As known from treatment trials in multiple sclerosis MRI can provide objective markers of the burden of disease such as the total lesion load which may be even more sensitive for determining the extent of brain damage than clinical measures. ${ }^{11}$ Furthermore, comparative analysis of lesion load as shown on various MRI sequences could serve to detect differences in the severity of tissue destruction between genotypes. Whereas multiple sclerosis lesions appear almost uniformly hyperintense on proton density and T2 weighted MRI only those lesions with more severe tissue loss are seen on T1 weighted scans. ${ }^{12}$ These lesions have been termed "black holes" and the grade of MRI lesion hypointensity was shown to correspond well with the magnitude of axonal damage, extracellular oedema, and the degree of demyelination or remyelination. ${ }^{13}{ }^{14}$

\section{Methods}

Over a period of 5 months we obtained blood samples for apoE genotyping from all patients admitted to our multiple sclerosis outpatient clinic who met Poser's criteria of laboratory supported or clinically definite multiple sclerosis. ${ }^{15}$ Eighty nine of these 149 patients had undergone MRI at our institution. Six patients had to be excluded because of incomplete data or limited quality of the examinations. This left 83 patients ( 47 women, 36 men) with a mean (SD) age of 35.7 (9.5) years (range 17-60 years). Seventy six patients had 
Table 1 Demographic and clinical findings in apoE genotype subgroups

\begin{tabular}{lllll}
\hline & All $(n=83)$ & $\varepsilon 2 / \varepsilon 3(n=11)$ & $\varepsilon 3 / \varepsilon 3(n=49)$ & $\varepsilon 3 / \varepsilon 4(n=19)$ \\
\hline Age (y) & $35.7(9.5)[34]$ & $38.7(3.0)[39]$ & $35.9(8.6)[35]$ & $34.0(9.6)[34]$ \\
Women (\%) & 58 & 55 & 59 & 58 \\
MS duration (months) & $90.5(80.1)[77]$ & $103.0(142.6)[77]$ & $91.0(71.3)[77]$ & $88.8(62.0)[82]$ \\
EDSS score & $1.9(1.6)[1.5]$ & $2.0(1.7)[2]$ & $1.8(1.4)[1.5]$ & $2.4(2.2)[1.5]$ \\
Relapses (number) & $6.2(4.6)[5.0]$ & $4.8(3.6)[4.0]$ & $6.2(3.9)[6.0]$ & $7.3(6.6)[5.5]$ \\
RR course (\%) & 90 & 100 & 96 & 79 \\
\hline
\end{tabular}

Data are given as mean (SD) [median].

EDSS=Expanded disability status scale; $R R=$ relapsing-remitting; $M S=$ multiple sclerosis.

a relapsing-remitting course of multiple sclerosis, five were in the secondary progressive phase and two patients had primary progressive multiple sclerosis. After informed consent, all patients were examined by one neurologist (SS-F) who recorded the pertinent demographic data, duration, and course of the disease, ${ }^{5}$ disease severity according to the Kurtzke expanded disability status scale, ${ }^{16}$ and the number of relapses. Relapses were defined as new or worsening neurological signs or symptoms with a duration of more than 24 hours. Retrospective data were confirmed by a review of the patient's charts.

All MRI examinations were performed on a 1.5 Tesla magnet (Philips Medical Systems, Eindhoven, The Netherlands) with a standard protocol using conventional spin echo sequences. This includes proton density and T2 weighted images (repetition time (TR))/echo time (TE): $2500 \mathrm{~ms} / 30$ and $90 \mathrm{~ms}$ ) and $\mathrm{T} 1$ weighted images (TR/TE $600 \mathrm{~ms} / 15 \mathrm{~ms}$ ) in the axial plane. The $\mathrm{T} 1$ weighted sequence was performed after injection of gadolinium-DTPA in a dosage of $0.1 \mathrm{mmol} / \mathrm{kg}$ bodyweight. The slice thickness was $5 \mathrm{~mm}$. The mean interval between MRI and the clinical examination at the time of blood sampling was 4.4 months.

Morphological analysis was performed on the PD weighted and T1 weighted images. Firstly, an experienced observer (FF) marked all lesions on hard copies. To improve reproducibility and concentrate on more marked tissue changes we used a stringent definition for black holes - that is, lesion hypointensity had to be at least between that of grey matter and $\mathrm{CSF}$ or lower. Areas of mild hypointensity were not considered in the $\mathrm{T} 1$ analysis. Lesion measurement was then performed by another investigator (CE) using "DispImage", an image processing software provided by David Plummer, University College, London, UK, which has been described in detail elsewhere. ${ }^{17}{ }^{18}$ Using this technique, the intraobserver variability was $1.2 \%$ for measurements of the total volume of hyperintensitiesthat is, the T2 lesion load (T2-LL) and 4\% for the entire "black hole" volume or T1 lesion load (T1-LL) in this study. This corresponds well with the intra-observer agreement reported by others. ${ }^{17}$ We also calculated a "black hole" ratio (BHR; (T1-LL/T2-LL) x 100) to indicate the proportion of more severe tissue destruction among multiple sclerosis lesions. All these analyses were performed without knowledge of the patients' clinical condition or genotype.

ApoE genotyping was done according to the method of Hixon and Vernier ${ }^{19}$ after extraction of high molecular weight DNA from peripheral blood.

STATISTICAL ANALYSIS

We used the statistical package for social sciences (SPSS/PC+) for data analysis. Categorical variables among the different apoE genotypes were compared by $\chi^{2}$ tests. We used Levene's test to confirm a normal distribution of continuous variables. Comparisons of continuous variables between genotypes were conducted by Student's $t$ test or with the non-parametric Mann-Whitney $U$ test in the absence of a normal distribution.

\section{Results}

The apoE genotype frequencies were as follows: $\varepsilon 2 / \varepsilon 2$ in two $(2.4 \%), \varepsilon 2 / \varepsilon 3$ in 11 $(13.3 \%), \varepsilon 3 / \varepsilon 3$ in $49(59 \%), \varepsilon 2 / \varepsilon 4$ in one (1.2\%), $\varepsilon 3 / \varepsilon 4$ in $19(22.9 \%)$, and $\varepsilon 4 / \varepsilon 4$ in one $(1.2 \%)$. This distribution of genotypes is in Hardy-Weinberg equilibrium. Consequently, the frequencies for the $\varepsilon 2, \varepsilon 3$, and $\varepsilon 4$ allele were 16,128 , and 22 .

Demographic and clinical data for the total cohort and the $\varepsilon 2 / \varepsilon 3, \varepsilon 3 / \varepsilon 3$, and $\varepsilon 3 / \varepsilon 4$ subsets at the time of blood sampling are shown in table 1. Patients with the $\varepsilon 3 / \varepsilon 4$ genotype tended to be younger and had the highest mean EDSS score and number of relapses despite the shortest disease duration. A relapsingremitting course was less frequent at examination in the presence of an $\varepsilon 3 / \varepsilon 4$ genotype. However, none of these clinical differences reached significance.

The clinical data at the time of MRI showed a distribution of genotypes which was similar to that seen at the time of blood sampling. Results of the MRI analysis are shown in table 2 .

Table 2 MRI findings in apoE genotype subgroups

\begin{tabular}{lllll}
\hline & All $(n=83)$ & $\varepsilon 2 / \varepsilon 3(n=11)$ & $\varepsilon 3 / \varepsilon 3(n=49)$ & $\varepsilon 3 / \varepsilon 4(n=19)$ \\
\hline T2 lesion load $\left(\mathrm{cm}^{3}\right)$ & $11.6(10.7)[8.7]$ & $13.3(9.5)[14.4]$ & $9.4(9.2)[5.6]$ & $16.0(14.0)[11.3]$ \\
T1 lesion load $\left(\mathrm{cm}^{3}\right)$ & $1.6(3.0)[0.4]$ & $1.6(2.4)[0.6]$ & $1.2(3.0)[0.1]$ & $2.6(3.3)[1.1]^{\star}$ \\
"Black hole" ratio (\%) & $9.6(12.5)[5.8]$ & $7.4(9.3)[6.2]$ & $8.4(13.3)[4.0]$ & $14.3(11.9)[12.4]^{\star}$ \\
Active scans (number) & 18 & 3 & 13 & $1 \dagger$ \\
\hline
\end{tabular}

Data are given as mean (SD) [median].

${ }^{\star} \varepsilon 3 / \varepsilon 4 v \varepsilon 3 / \varepsilon 3, \mathrm{p}<0.02$ (Mann-Whitney $U$ test).

$\dagger \varepsilon 3 / \varepsilon 4$ v $\varepsilon 3 / \varepsilon 3, \mathrm{p}<0.05$ ( $\chi^{2}$ test). 

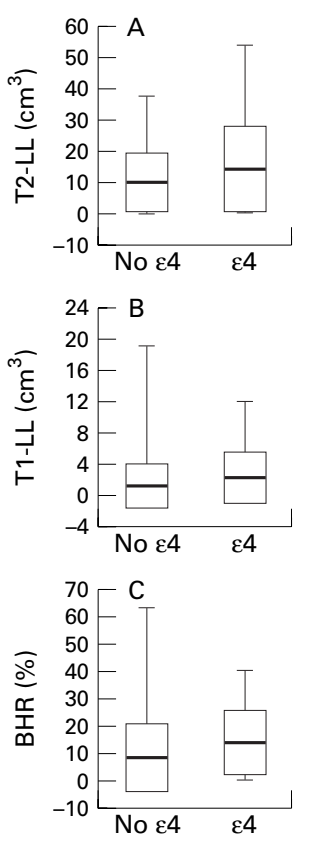

Box plots of MRI measures of multiple sclerosis lesion load in patients with and without at least one apoE $\varepsilon 4$ allele.
Patients with the $\varepsilon 3 / \varepsilon 4$ genotype had the greatest T2-LL. Differences in the T1-LL were even more pronounced and statistically significant between the $\varepsilon 3 / \varepsilon 4$ and the $\varepsilon 3 / \varepsilon 3$ genotype subgroups $(p=0.018)$. Moreover, the black hole ratio was almost twice as high in patients with the $\varepsilon 3 / \varepsilon 4$ genotype than in the other genotypes. Again this difference reached significance between $\varepsilon 3 / \varepsilon 4$ and $\varepsilon 3 / \varepsilon 3$ subsets $(\mathrm{p}=0.014)$. Interestingly, active scans as indicated by the presence of contrast enhancing lesions were noted less often in $\varepsilon 3 / \varepsilon 4$ patients, however $(p=0.048)$. Comparison between carriers of at least one $\varepsilon 4$ allele to the remainder of the study population confirmed a trend towards a higher T2-LL (15.1 (SD 13.7) v 10.4 (SD 9.2) $\mathrm{cm}^{3}$, $\mathrm{p}=0.24)$ and a significantly greater T1-LL $(2.4$ $\left.(\mathrm{SD} 3.2) v 1.3(\mathrm{SD} 2.8) \mathrm{cm}^{3}, \mathrm{p}=0.044\right)$ and black hole ratio (13.3 (SD 11.8) v 8.3 (SD $12.5) \%, p=0.024$ ) in $\varepsilon 4$ patients (figure $\mathrm{A}-\mathrm{C}$ ).

\section{Discussion}

Earlier studies on the apoE genotype in multiple sclerosis have dealt primarily with allele frequencies and found no significant differences in the distribution of genotypes between patients with multiple sclerosis and the normal population. ${ }^{9021}$ However, a more aggressive course of multiple sclerosis in patients with the apoE $\varepsilon 4$ allele has been suggested by different investigators. $^{9}{ }^{10}$ This was not confirmed in a recent study on 361 patients with clinically definite multiple sclerosis. ${ }^{22}$ Morphological findings of the present study add to this controversy.

Using MRI in a cross sectional study design we found the highest lesion load in patients with multiple sclerosis with the apoE $\varepsilon 3 / \varepsilon 4$ genotype. This difference was more pronounced for the T1-LL than the T2-LL and was significant between the $\varepsilon 3 / \varepsilon 4$ and the $\varepsilon 3 / \varepsilon 3$ subgroups. In parallel there was also a significant difference between these genotypes for the relative proportion of "black holes". The black hole ratio of patients with the $\varepsilon 3 / \varepsilon 4$ genotype was almost twice that of patients with an $\varepsilon 2 / \varepsilon 3$ or $\varepsilon 3 / \varepsilon 3$ genotype. Similar and significant differences were noted when comparing all patients with at least one $\varepsilon 4$ allele to the remainder of the study population. Histopathological studies have shown that black holes represent multiple sclerosis lesions with more severe tissue destruction. ${ }^{13}{ }^{14}$ Furthermore, using MR spectroscopy a direct correlation was found between the extent of hypointensity of multiple sclerosis lesions on $\mathrm{T} 1$ weighted images and the relative reduction in $\mathrm{N}$-acetylaspartate, which is considered a marker for axonal density. ${ }^{23}$ Therefore, our findings of a higher T1-LL and higher black hole ratio can be viewed as indirect evidence for a less favourable course of multiple sclerosis in patients carrying the $\varepsilon 4$ allele. Clinically our patients with an apoE $\varepsilon 3 / \varepsilon 4$ genotype also tended to have more aggressive disease with greater disability and a higher number of relapses experienced in a shorter period of time. These differences did not reach significance but are in line with the recent finding of a faster increase of disability in apoE $\varepsilon 4$ carriers during 2 years of treatment with glatiramer acetate. $^{24}$

Whether a higher proportion of T1-LL in apoE $\varepsilon 4$ patients is due to more aggressive disease with extensive demyelination or axonal loss within acute plaques or a consequence of less efficient repair thereafter cannot yet be determined. More active multiple sclerosis in apoE $\varepsilon 4$ carriers could be another explanation for our findings. This would be supported by their overall larger T2-LL and a somewhat higher number of relapses. However, a significantly lower frequency of active scans in patients with the apoE $\varepsilon 3 / \varepsilon 4$ genotype argues against this assumption although this finding certainly suffers from the cross sectional design of our study and the relatively few patients examined. For these reasons and because the proportion of patients with any treatment was similar between genotype subgroups we also did not attempt to consider the possible influence of different treatments at the time of the MRI examination.

These preliminary results provide morphological support for a negative effect of the apoE $\varepsilon 4$ allele on the course of multiple sclerosis. They also illustrate the capability of MRI to detect small differences between patient groups which clinically would have gone undetected. Although these data cannot serve to explain the reasons for divergent findings in previous clinical studies they indicate the need for further investigation of the the role of the apoE genotype in multiple sclerosis. Such efforts may also take advantage of the sensitivity and objective nature of MRI findings in the future. This work was supported by the Austrian MS Society. SR is
supported by the European Charcot Foundation and the Hertie Foundation.

1 Poser C. The epidemiology of multiple sclerosis. A general Poser C. The epidemiology of multiple sclerosis.

2 Sadovnick AD, Ebers GC, Dyment DA, et al. Evidence for genetic basis of multiple sclerosis. The Canadian Collaborative Study Group. Lancet 1996;347:1728-30.

3 Sawcer S, Goodfellow PN, Compston A. The genetic analysis of multiple sclerosis. Trends Genet 1997;13:234-9.

4 Weinshenker BG, Bass B, Rice GP, et al. The natural history of multiple sclerosis: a geographically based study. I: Clinical course and disability. Brain 1989;112:113-46.

5 Lublin FD, Reingold SC. Defining the clinical course of multiple sclerosis: results of an international survey. Neurology 1996;46:907-11.

6 Mahley RW. Apolipoprotein E: cholesterol transport protein with expanding role in cell biology. Science 1988;240:62230

7 Nathan BP, Bellosta S, Sanan DA, et al. Differential effects of apolipoprotein E3 and E4 on neuronal growth in vitro. Science 1994:264:850-2

8 Boyles JK, Zoellner CD, Anderson LJ, et al. A role for apolipoprotein E, apolipoprotein A-I, and low density lipoprotein receptors in cholesterol transport during regeneration and remyelinisation of the rat sciatic nerve. 7 Clin Invest 1989;83:1015-31.

9 Evangelou N, Jackson M, Beeson D, et al. Association of the APOE $\varepsilon 4$ allele with disease activity in multiple sclerosis. $\mathcal{F}$ Neurol Neurosurg Psychiatry 1999;67:203-5.

10 Høgh P, Blinkenberg M, Jørgensen OS, et al. Apolipoprotein $\mathrm{E}$ (APOE) and multiple sclerosis: impact of the epsilon-4 allele on suceptibility, clinical type and progression rate [abstract]. Eur 7 Neurol 1998;5(suppl 3):121

11 Miller DH, Grossman RI, Reingold SC, et al. The role of magnetic resonance techniques in understanding and managing multiple sclerosis. Brain 1998;121:3-24.

12 van Walderveen MAA, Barkhof F, Hommes OR, et al. Correlating MRI and clinical disease activity in multiple sclerosis: relevance of hypointense lesions on short-TR / short-TE (T1-weighted) spin-echo images. Neurology 1995;45:1684-90.

13 Brück W, Bitsch A, Kolenda H, et al. Inflammatory central nervous system demyelination. Correlation of magnetic 
resonance imaging findings with lesion pathology. Ann Neurol 1997;42:783-93.

14 van Walderveen MAA, Kamphorst W, Scheltens P, et al. Histopathologic correlate of hypointense lesions on T1 weighted spin-echo MRI in multiple sclerosis. Neurology 1998; 50:1282-8.

15 Poser CM, Paty DW, Scheinberg L, et al. New diagnostic criteria for multiple sclerosis: guidelines for research protocols. Ann Neurol 1983;13:227-31.

16 Kurtzke JF. Rating neurologic impairment in multiple sclerosis: an expanded disability status scale (EDSS). Neurology 1983;33:1444-52.

17 Rovaris M, Filippi M, Calori G, et al. Intra-observer reproducibility in measuring new putative MR markers of demyelination and axonal loss in multiple sclerosis: a comparison with conventional T2-weighted images. F Neurol 1997; 244:266-70.

18 Filippi M, Gawne-Cain ML, Gasperini C, et al. Effect of training and different measurement strategies on the reproducibility of brain MRI lesion load measurements in multiple sclerosis. Neurology 1998;50:238-44.
19 Hixon JE, Vernier DT. Restriction isotyping of human apolipoprotein $\mathrm{E}$ by gene amplification and cleavage with Hhal. F Lipid Res 1990;31:545-8.

20 Rubinsztein DC, Hanlon CS, Irving RM, et al. Apo E genotypes in multiple sclerosis, Parkinson's disease, schwannomas and late-onset Alzheimer's disease. Mol Cell Probes 1994;8:519-25.

21 Gervais A, Gaillard O, Plassart E, et al. Apolipoprotein E polymorphism in multiple sclerosis. Ann Clin Biochem 1998;35:135-6.

22 Weatherby SJM, Mann CLA, Davies MB, et al. Polymorphisms of apolipoprotein E: outcome and susceptibility of multiple sclerosis. Mult Scler 2000;6:32-6.

23 van Walderveen MAA, Barkhof F, Pouwels PJW, et al. Neuronal damage in $\mathrm{T}_{1}$-hypointense multiple sclerosis lesions demonstrated in vivo using proton magnetic resonance spectroscopy. Ann Neurol 1999;46:79-87.

24 Chapman J, Sylantiev C, Nisipeanu P, et al. Preliminary observations on APOE $\& 4$ allele and progression of disability in multiple sclerosis. Arch Neurol 1999;56:1484-7. 